\title{
Application of Koutecký-Levich Method to the Analysis of Steady State Voltammograms with Ultramicroelectrodes
}

Jiyeon Kim and Allen J. Bard

\author{
Supporting information
}

Table of Contents

Page

The derivation for irreversible, one-step one electron transfer reduction reaction

(Equations S1 - S6 and Figure S1)

The derivation for quasi-reversible, one step one electron transfer reduction reaction

S6

(Equations S7 - S10 and Figure S2)

The derivation for quasi-reversible, one-step one electron transfers oxidation reaction (Equations S11 - S15)

The derivation for reversible, one-step one electron transfer reaction

S8

(Equation S16)

Applicable potential range for K-L analysis (Table S1, S2, Figure S3)

Finite Element Simulations (Figure S4 and Table S3) 
We consider a generic one step electrochemical reaction,

$$
\mathrm{O}+\mathrm{ne}^{-} \underset{k_{b}}{\stackrel{k_{f}}{\rightleftarrows}} \mathrm{R}
$$

where a redox mediator $\mathrm{O}$ is reduced to $\mathrm{R}$ at a UME. In this case, the steady-state current is governed by the rates of two competitive processes.

(a) The mass transfer of $\mathrm{O}$ species from the bulk solution to near a UME surface.

(b) The ET from the UME surface to species, O.

\section{1) The derivation for irreversible, one-step one electron transfer reduction reaction.}

We treat the overall process as a sum of the reciprocals of the two competing processes of mass transfer and electron transfer, so that the total current density, $j$ can be expressed as $\mathrm{S} 1$

$$
\frac{1}{j}=\frac{1}{j_{m t}}+\frac{1}{j_{e t}}
$$

where, $j_{e t}$ and $j_{m t}$ are the limiting current densities for heterogeneous kinetics at a UME surface and mass transfer of $\mathrm{O}$ to a UME surface, respectively. A mass transfer limited current density, $j_{m t}$ can be written:

$$
j_{m t}=F m_{O} C_{O}^{*}
$$

where $F$ is the Faraday constant, $C_{O}^{*}$ is the bulk concentration of $\mathrm{O}$ in solution. $m_{O}$ is a mass transfer coefficient, expressed in terms of the size and the shape of UMEs, e.g., $m_{O}=4 D / \pi r_{0}$ for a 
disk UME, $m_{O}=D / r_{0}$ for a spherical electrode, where $r_{0}$ is the radius of each geometry and $D$ is diffusion coefficient, which is well known from the theory of UMEs.S2

'Here, we use the Butler-Volmer model to treat heterogeneous kinetic currents and assume $\mathrm{n}=1 .{ }^{\mathrm{S} 2}$ For an irreversible ET reaction (negligible backward reaction in equation $\mathrm{S} 1$ ), $j$ is given bys2

$$
\begin{gathered}
j=F k_{f} C_{O(y=0)} \\
k_{f}=k^{0} e^{-\frac{\alpha F}{R T}\left(E-E^{o \prime}\right)} \\
C_{O(y=0)}=C_{O}^{*}\left(1-\frac{j}{j_{m t}}\right)
\end{gathered}
$$

where $k_{f}$ is the forward reaction rates and $k^{0}$ and $E^{0^{\prime}}$ are the heterogeneous standard reaction rate constant and the standard reduction potential, respectively. $\alpha$ is the transfer coefficient and $F$ is the Faraday constant, $96485 \mathrm{C} / \mathrm{mol}, \mathrm{R}$ is the gas constant, and $\mathrm{T}$ is the temperature. $k_{f}$ (or $k_{b}$ ) is written according to the Butler-Volmer model. $C_{O(y=0)}$ is the concentration of $\mathrm{O}$ at the electrode surface. Replacing the expression of $C_{O(y=0)}$ to equation S4 yields,

$$
j=F k_{f} C_{O}^{*}\left(1-\frac{j}{j_{m t}}\right)
$$

Defining a kinetic limiting current density, $j_{e t}=F k^{0} C_{O}^{*} e^{-\frac{\alpha F}{R T}\left(E-E^{0^{\prime}}\right)}$ with $b=e^{\frac{F}{R T}\left(E-E^{0^{\prime}}\right)}$, and rearranging obtains,

$$
\frac{1}{j}=\frac{1}{F m_{O} C_{O}^{*}}+\frac{b^{\alpha}}{F k^{0} C_{O}^{*}}
$$


which is directly analogous to equation $\mathrm{S} 2$, and of the $\mathrm{K}-\mathrm{L}$ treatment of an irreversible reaction at a rotating disk electrode as shown in our previous work.S1 Thus, it includes contributions from both the mass transfer and heterogeneous kinetics.

Instead of varying angular rotation rates of an electrode to alter the mass transfer rate, $m_{O}$ in the classical K-L treatment, the size of UMEs can be varied to modulate $m_{O}$. So, one can obtain the steady state voltammograms with UMEs of varying sizes, and subsequently, a plot of $1 / j$ vs. $1 /\left(m_{O} C_{O}{ }^{*}\right)$ at different potentials can be constructed from the obtained voltammograms. These plots should be linear with a slope of $1 / F$. Importantly, an extrapolation to $1 /\left(m_{O} C_{O}{ }^{*}\right) \rightarrow 0$ allows for measuring a y-intercept, $b^{\alpha} / F k^{0} C_{O}{ }^{*}$ thus determining kinetic parameters for the ET reaction. At given different potentials, expressed as $\left(E-E^{0^{\prime}}\right)$, a set of $1 / j$ vs. $1 /\left(m_{O} C_{O}{ }^{*}\right)$ can be plotted, where the rate of ET is sufficiently slow to act as a limiting factor, or where ET is rapid in the limiting current regime. The less cathodic potential would result in the larger $b^{\alpha}$, thus the larger y-intercept. Consequently, several y-intercepts can be obtained at different potentials, thereby the kinetic parameters $\alpha$ and $k^{0}$ can be determined using Butler-Volmer model.

We confirmed our theoretical approach by finite element analysis with COMSOL MULTIPHYSICS v 4.2a. Voltammograms were simulated for one step, one electron reduction of oxidized species, $\mathrm{O}$, where $E^{0}, C_{O}{ }^{*}, C_{R}{ }^{*}, E_{e q}$ and $\alpha$ are $0.3 \mathrm{~V}, 1 \mathrm{mM}, 0.01 \mathrm{mM}, 0.42 \mathrm{~V}$ and 0.5 , respectively for Pt disk UMEs with radii of $150 \mathrm{~nm}, 600 \mathrm{~nm}, 2.5$ and $5 \mu \mathrm{m}$. Diffusion coefficients, $D$ of oxidized and reduced redox molecules were assumed to be same as $7.4 \times 10^{-6}$ $\mathrm{cm}^{2} / \mathrm{s}$. For an irreversible reaction with $k^{0}=0.04 \mathrm{~cm} / \mathrm{s}$, a set of voltammograms were simulated as presented in Figure S1(a), which are normalized with the respective steady state currents to show differences in curve shapes. Clearly, the half wave potential, $E_{1 / 2}$, is shifted by c.a. $200 \mathrm{mV}$ 
as the electrodes become smaller, from $5 \mu \mathrm{m}$ to $150 \mathrm{~nm}$. Such a sluggish current response for smaller electrodes is attributed to more kinetically limited ET due to more efficient mass transfer. From simulated voltammograms with different sizes of UMEs, current densities $(j)$ at different potentials, e.g. $E-E^{0^{\prime}}=-0.40,-0.12,-0.06$ and $-0.00 \mathrm{~V}$ were chosen to subsequently replot $1 / j$ vs. $1 /\left(m_{O} C_{O}{ }^{*}\right)$ (shown in Figure S1(b)). As predicted, a set of linear plots with a constant slope of $1 / F$ was obtained. At $E-E^{0^{\prime}}=-0.40 \mathrm{~V}$, the electrochemical reaction is primarily limited by the mass transfer, so that a plot of $1 / j$ vs. $1 /\left(m_{O} C_{O}{ }^{*}\right)$ obtained at $E-E^{0^{\prime}}=-0.40 \mathrm{~V}$ intersects the origin. As expected, a less cathodic potential brought a larger $y$-intercept in the plot of $1 / j$ vs. $1 /\left(m_{O} C_{O}^{*}\right)$

(a)

(b)
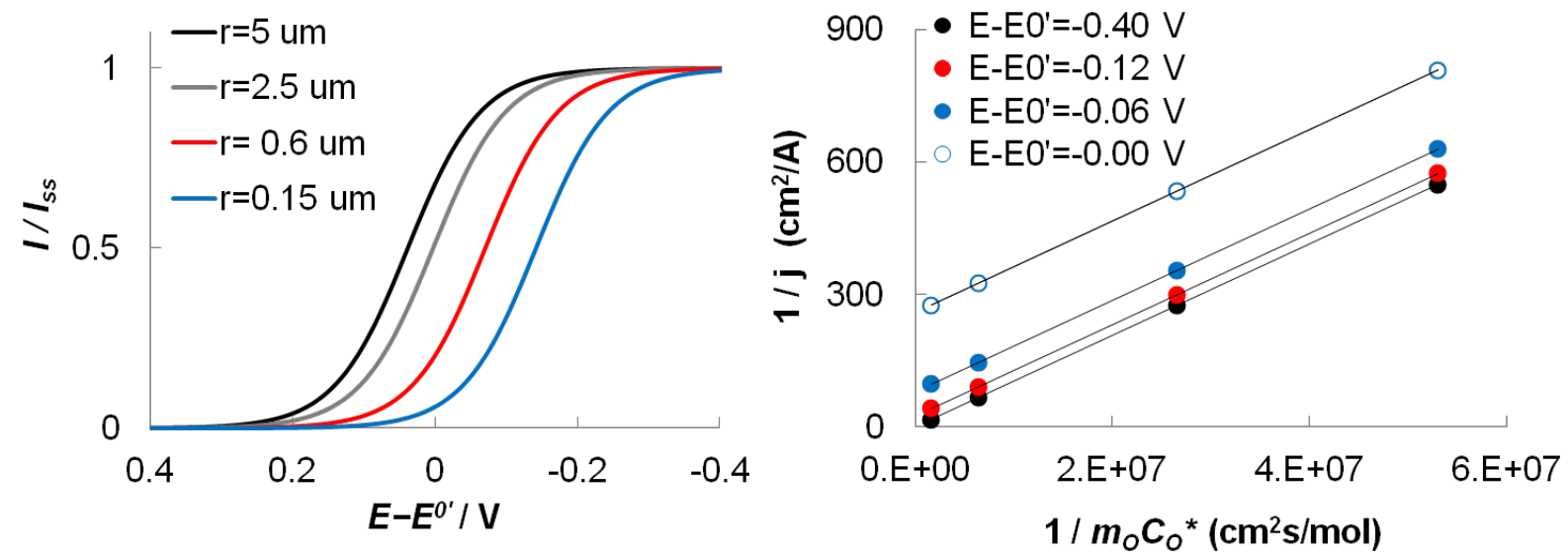

Figure S1. (a) Simulated steady state voltammograms by COMSOL MUTIPHYSICS v.4.2 for an irreversible one electron reduction reaction with $k^{0}=0.04 \mathrm{~cm} / \mathrm{s}$ and $E^{0^{\prime}}, C_{O}{ }^{*}, C_{R}{ }^{*}, E_{e q}$ and $\alpha$ are $0.3 \mathrm{~V}, 1 \mathrm{mM}, 0.01 \mathrm{mM}, 0.42 \mathrm{~V}$ and 0.5 , respectively with various radii of Pt disk UMEs, 150 $\mathrm{nm}, 600 \mathrm{~nm}, 2.5$ and $5 \mu \mathrm{m}$. Diffusion coefficient and concentration of $\mathrm{O}$ species are $7.4 \times 10^{-6}$ $\mathrm{cm}^{2} / \mathrm{s}$ and $1 \mathrm{mM}$, thus $m_{O}=0.019,0.038,0.16$ and $0.63 \mathrm{~cm} / \mathrm{s}$, respectively. All currents are normalized by their respective steady state currents. (b) a set of linear plots of $1 / j \mathrm{vs} .1 / \mathrm{m}_{O} C_{O}{ }^{*}$ at given potentials $E-E^{0^{\prime}}=-0.40,-0.12,-0.06,-0.00 \mathrm{~V}$ from voltammograms in Figure S1(a). A slope and y intercepts are $1 / F$ and $1 / F C_{O}{ }^{*} k_{f}$ (i.e., $b^{\alpha} / F C_{O}{ }^{*} k^{0}$ ), respectively. A less cathodic potential leads to a larger $y$-intercept. 


\section{2) The derivation for quasi-reversible, one step one electron transfer reduction reaction.}

The steady state current density for a quasi-reversible case is expressed as, $\mathrm{S} 2$

$$
\begin{gathered}
j=F\left(k_{f} C_{O(y=0)}-k_{b} C_{R(y=0)}\right) \\
k_{f}=k^{0} e^{-\frac{\alpha F}{R T}\left(E-E^{o \prime}\right)}, k_{b}=k^{0} e^{\frac{(1-\alpha) F}{R T}\left(E-E^{o \prime}\right)} \\
C_{O(y=0)}=C_{O}^{*}\left(1-\frac{j}{j_{m t, c}}\right), C_{R(y=0)}=C_{R}^{*}\left(1-\frac{j}{j_{m t, a}}\right) \\
j_{m t, c}=F m_{O} C_{O}^{*}, j_{m t, a}=-F m_{R} C_{R}^{*}
\end{gathered}
$$

where, $k_{b}$ is the backward reaction rate, $C_{R}^{*}$ is the bulk concentration of $\mathrm{R}$ in solution, $C_{R(y=0)}$ is the concentration of $\mathrm{R}$ at the electrode surface, and other parameters are the same as the irreversible ET case written above. By defining the exchange current density, $j_{0}=F k^{0} C_{0}^{*} e^{\frac{-\alpha F}{R T}\left(E_{e q}-E^{0^{\prime}}\right)}=F k^{0} C_{0}^{* 1-\alpha} C_{R}^{* \alpha} \quad$ with $\quad b=e^{\frac{F}{R T}\left(E-E_{e q}\right)} \quad$ and $\quad E_{e q}=E^{0^{\prime}}+$ $\frac{R T}{F} \ln \left(\frac{C_{O}^{*}}{C_{R}^{*}}\right)$, rearranging equation $\mathrm{S} 4$ yields,

$$
\begin{gathered}
j=j_{0}\left(\left(\frac{C_{O(y=0)}}{C_{O}^{*}}\right) b^{-\alpha}-\left(\frac{C_{R(y=0)}}{C_{R}^{*}}\right) b^{1-\alpha}\right) \\
j=j_{0}\left(\left(1-\frac{j}{j_{m t, c}}\right) b^{-\alpha}-\left(1-\frac{j}{j_{m t, a}}\right) b^{1-\alpha}\right)
\end{gathered}
$$

If $C^{*}=C_{O}^{*}$, the concentration ratio, $C_{O}^{*} / C_{R}^{*}=p$, $m_{O}=m$, and the mass transfer coefficient ratio, $m_{O} / m_{R}$ is $q$, accordingly, equation S8 can be written as 


$$
\begin{gathered}
\frac{1}{j}=\frac{b^{\alpha}}{1-b}\left(\frac{1}{j_{0}}+\frac{1}{F m}\left(\frac{b^{-\alpha}}{C_{O}^{*}}+\frac{q b^{1-\alpha}}{C_{R}^{*}}\right)\right) \\
\frac{1}{j}=\frac{b^{\alpha}}{1-b}\left(\frac{1}{F k^{0} C^{*}(p)^{-\alpha}}+\frac{1}{F m C^{*}}\left(b^{-\alpha}+p q b^{1-\alpha}\right)\right) \\
\frac{1}{j}=\frac{1}{F k^{0} C^{*}} \frac{(p b)^{\alpha}}{(1-b)}+\frac{1}{F m C^{*}}\left(\frac{1+p q b}{1-b}\right)
\end{gathered}
$$

which is directly analogous to equation S2 including the contribution of the kinetic and the mass transfer terms, thus a similar form to K-L relationship

We further confirmed our theoretical approach by finite element analysis with COMSOL MULTIPHYSICS v 4.2a. Voltammograms were simulated for one step, one electron reduction of oxidized species, $\mathrm{O}$, where $E^{0^{\prime}}, C_{O}{ }^{*}, C_{R}{ }^{*}, E_{e q}$ and $\alpha$ are $0.3 \mathrm{~V}, 1 \mathrm{mM}, 0.01 \mathrm{mM}, 0.42 \mathrm{~V}$ and 0.5 , respectively for Pt disk UMEs with radii of $150 \mathrm{~nm}, 600 \mathrm{~nm}, 2.5$ and $5 \mu \mathrm{m}$. Diffusion coefficients, $D$ of oxidized and reduced redox molecules were assumed to be same as $7.4 \times 10^{-6}$ $\mathrm{cm}^{2} / \mathrm{s}$. For the slow quasi-reversible reaction with $k^{0}=4 \times 10^{-4} \mathrm{~cm} / \mathrm{s}$, a series of steady state voltammograms were simulated for Pt disk UMEs with radii from $150 \mathrm{~nm}$ to $5 \mu \mathrm{m}$ (Figure S2(a)) and corresponding plots of $1 / j$ vs. $1 /\left(m_{O} C_{O}{ }^{*}\right)$ were obtained at different given potentials as depicted in Figure S2(b). Notably, large y-intercepts can be seen compared to a quasi-reversible reaction with $k^{0}=0.04 \mathrm{~cm} / \mathrm{s}$, while a slope is maintained at $1 / F$. In addition, a very fast quasireversible case showing almost Nernstian behavior was studied with $k^{0}=4 \mathrm{~cm} / \mathrm{s}$ (voltammograms in Figure S2(c)). A linear plot of $1 / j$ vs. $1 /\left(m_{O} C_{O}{ }^{*}\right)$ obtained at a mass transfer limited potential intersects the origin (Figure S2(d)). At any given potentials, the deviation in the y-axis intercept was too small to be significantly considered, hence no discernible kinetic term could be measured. 
(a)

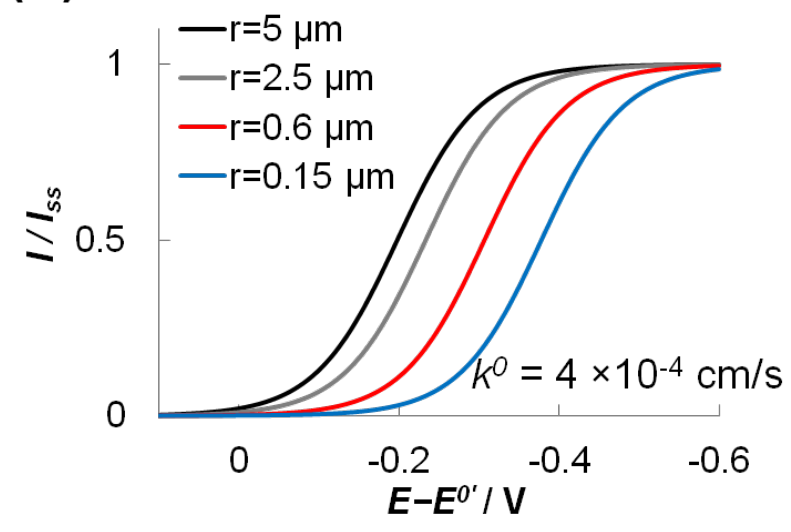

(b)

3000

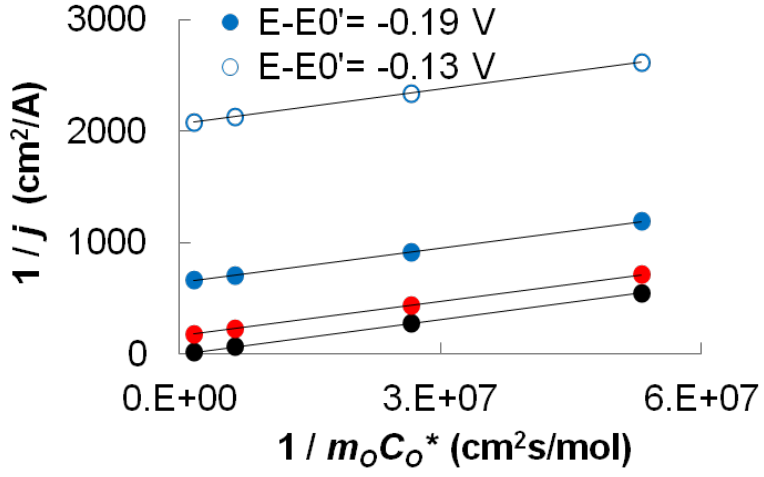

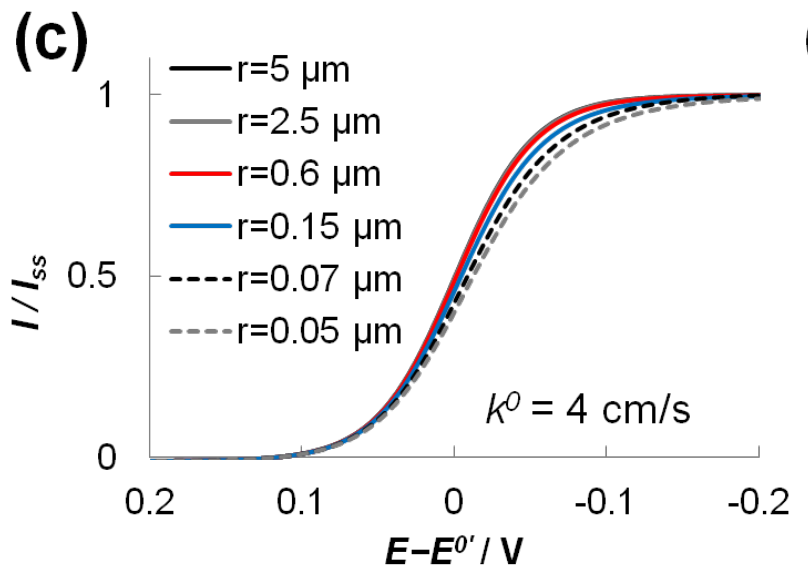

(d)

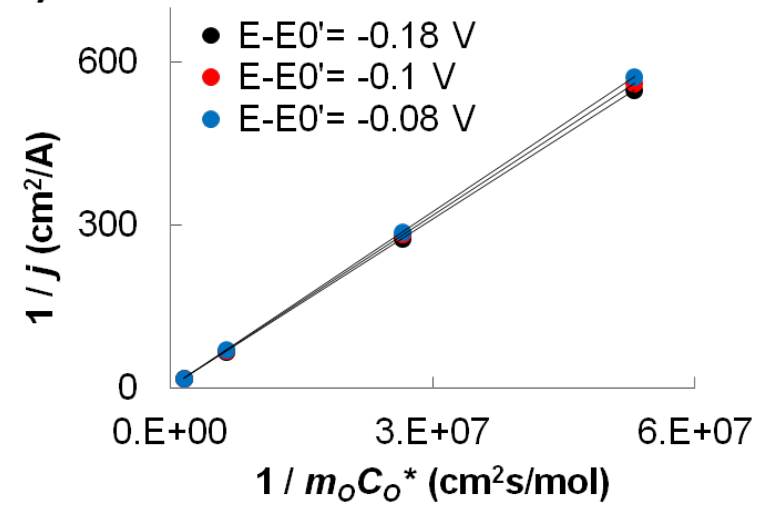

Figure S2. (a) and (c) Simulated steady state voltammograms for a quasi-reversible one electron reduction reaction, with $k^{0}=4 \times 10^{-4} \mathrm{~cm} / \mathrm{s}$ and $k^{0}=4 \mathrm{~cm} / \mathrm{s}$, respectively. $E^{0^{\prime}}, C_{O}{ }^{*}, C_{R}{ }^{*}, E_{e q}$ and $\alpha$ are $0.3 \mathrm{~V}, 1 \mathrm{mM}, 0.01 \mathrm{mM}, 0.42 \mathrm{~V}$ and 0.5 , respectively with various radii of Pt disk UMEs, 150 $\mathrm{nm}, 600 \mathrm{~nm}, 2.5$ and $5 \mu \mathrm{m}$ for (a) and $50 \mathrm{~nm}, 70 \mathrm{~nm}, 150 \mathrm{~nm}, 600 \mathrm{~nm}, 2.5$ and $5 \mu \mathrm{m}$ for (c). All currents in voltammograms are normalized by their respective steady state currents. (b) and (d) Corresponding plots of $1 / j$ vs. $1 / m_{O} C_{O}{ }^{*}$ to (a) and (c), respectively. Slopes of all lines are $1 / F$. No discernible deviation of y-intercept from origin was seen with at any given potentials for $k^{0}=$ $4 \mathrm{~cm} / \mathrm{s}$.

3) The derivation for quasi-reversible, one-step one electron transfer oxidation reaction.

$$
\mathrm{R}-\mathrm{e}-\underset{k_{b}}{\stackrel{k_{f}}{\rightleftarrows}} \mathrm{O}
$$


The steady state current can be written as, $\mathrm{S} 2$

$$
\begin{gathered}
j=F\left(k_{f} C_{R(y=0)}-k_{b} C_{O(y=0)}\right) \\
k_{f}=k^{0} e^{\frac{\alpha F}{R T}\left(E-E^{o \prime}\right)}, k_{b}=k^{0} e^{\frac{-(1-\alpha) F}{R T}\left(E-E^{o \prime}\right)} \\
C_{O(y=0)}=C_{O}^{*}\left(1-\frac{j}{j_{m t, c}}\right), C_{R(y=0)}=C_{R}^{*}\left(1-\frac{j}{j_{m t, a}}\right) \\
j_{m t, a}=F m_{R} C_{R}^{*}, \quad j_{m t, c}=-F m_{O} C_{O}^{*}
\end{gathered}
$$

where, $k_{f}$ and $k_{b}$ is the forward and backward reaction rates, $C_{O}{ }^{*}$ and $C_{R}^{*}$ are the bulk concentration of $\mathrm{O}$ and $\mathrm{R}$ in solution, $C_{R(y=0)}$ and $C_{R(y=0)}$ are the concentration of $\mathrm{O}$ and $\mathrm{R}$ at the electrode surface, and other parameters are same as written in main text. By defining the exchange current density, $j_{0}=F k^{0} C_{R}^{*} e^{\frac{(1-\alpha) F}{R T}\left(E_{e q}-E^{0^{\prime}}\right)}=F k^{0} C_{R}^{* 1-\alpha} C_{O}^{* \alpha}$ with $b=e^{\frac{F}{R T}\left(E-E_{e q}\right)}$ and $E_{e q}=E^{0^{\prime}}+\frac{R T}{F} \ln \left(\frac{C_{O}^{*}}{C_{R}^{*}}\right)$, rearranging equation $\mathrm{S} 12$ yields,

$$
\begin{aligned}
& j=j_{0}\left(\left(\frac{C_{R(y=0)}}{C_{R}^{*}}\right) b^{\alpha}-\left(\frac{C_{O(y=0)}}{C_{O}^{*}}\right) b^{-(1-\alpha)}\right) \\
& j=j_{0}\left(\left(1-\frac{j}{j_{m t, a}}\right) b^{\alpha}-\left(1-\frac{j}{j_{m t, c}}\right) b^{-(1-\alpha)}\right)
\end{aligned}
$$

If $C^{*}=C_{R}^{*}$, the concentration ratio, $C_{R}^{*} / C_{O}^{*}=p$, $m_{R}=m$, and the mass transfer coefficient ratio, $m_{R} / m_{O}$ is $q .$, accordingly, equation $\mathrm{S} 13$ can be written as 


$$
\begin{aligned}
& \frac{1}{j}=\frac{b^{-\alpha}}{\left(1-\frac{1}{b}\right)}\left(\frac{1}{j_{0}}+\frac{1}{F m}\left(\frac{b^{\alpha}}{C_{R}^{*}}+\frac{q b^{\alpha-1}}{C_{O}^{*}}\right)\right) \\
& \frac{1}{j}=\frac{b^{-\alpha}}{\left(1-\frac{1}{b}\right)}\left(\frac{1}{F k^{0} C^{*}(p)^{-\alpha}}+\frac{1}{F m C^{*}}\left(b^{\alpha}+p q b^{\alpha-1}\right)\right) \\
& \frac{1}{j}=\frac{(p)^{\alpha}}{F k^{0} C^{*} b^{\alpha-1}(b-1)}+\frac{1}{F m C^{*}}\left(\frac{b+p q}{b-1}\right)
\end{aligned}
$$

which is directly analogous to equation S2.

\section{4) The derivation for reversible, one-step one electron transfer reaction.}

For a reversible ET case, the contribution of the kinetic term, $1 / j_{e t}$ to overall $1 / j$ can be neglected due to $k^{0}$ being very large, thus:

$$
\frac{1}{j}=\frac{1}{F m_{O} C_{O}^{*}}
$$

For this case, $1 / j$ only varies as a function of $1 /\left(m_{O} C_{O}{ }^{*}\right)$. The resulting plot intersects origin with a slope of $1 / F$. In that sense, any deviation of the $y$-axis intercept from 0 in the linear plot of $1 / j$ vs. $1 /\left(m_{O} C_{O}{ }^{*}\right)$ implies a kinetic limitation in the ET reaction.

\section{5) Applicable potential range for $K-L$ analysis}

In this section, we discussed the dependence of $b$ values on the potentials and the corresponding change of a slope in K-L plot. 
For a quasi reversible one electron transfer reduction, a linear K-L plot with a slope, $\frac{(1+p q b)}{F(1-b)}$ can be obtained if $1 / j$ is plotted against $1 / m C^{*}$ (see section (2)). An extrapolation to $1 /\left(m C^{*}\right) \rightarrow 0$ allows for the determination of kinetic parameters for the electron transfer reaction from the y-intercept, $\frac{1}{F k^{0} C^{*}} \frac{(p b)^{\alpha}}{(1-b)}$. Especially, in the range of potential, $E-E^{0^{\prime}} \leq-60 \mathrm{mV}$, a constant slope of $1 / F$ is expected from obtained linear plots due to a negligibly small $b$ (Table S1.). Resultantly, parallel linear plots with different y-intercepts can be yielded at given different potentials. In other ranges of potential, $b$ becomes significant, so that the slope becomes variable and deviated from $1 / F$. In that sense, our approach for a quasi-reversible case would be more applicable in the potential range of $E-E^{0^{\prime}} \leq-60 \mathrm{mV}$ for one electron reduction reaction, where a slope of a linear plot maintains $1 / F$ independent with applied $E$.

Similarly, for a quasi-reversible oxidation reaction with one step, one electron transfer, one can derive the overall current density equations in the same way to get the slope, $\frac{(b+p q)}{F(b-1)}$ and y-intercept, $\frac{(p)^{\alpha}}{F k^{0} C^{*} b^{\alpha-1}(b-1)}$, when $C^{*}=C_{R}^{*}$, the concentration ratio, $C_{R}{ }^{*} / C_{O}{ }^{*}$ is $p, m_{R}=m$, and the mass transfer coefficient ratio, $m_{R} / m_{O}$ is $q$ (see section (3)). Analogously, our approach is more applicable in the potential range of $E^{-} E^{0^{\prime}} \geq 60 \mathrm{mV}$, where a slope of a linear plot maintains $1 / F$ independent with applied $E$ due to a dominantly large $b$ (Table S2.). 


\begin{tabular}{ccccc}
\hline $\mathbf{E}-\mathbf{E}^{\mathbf{0}^{6}} / \mathbf{V}$ & $\mathbf{E}-\mathbf{E}_{\mathbf{e q}} / \mathbf{V}$ & $\mathbf{b}$ & $\mathbf{( 1 + 1 0 0 b}) /(\mathbf{1}-\mathbf{b})$ & slope \\
\hline $\mathbf{- 0 . 3}$ & -0.418 & $8.43 \times 10^{-8}$ & 1.00 & $1 / F$ \\
$\mathbf{- 0 . 2}$ & -0.318 & $4.14 \times 10^{-6}$ & 1.00 & $1 / F$ \\
$\mathbf{- 0 . 1}$ & -0.218 & $2.03 \times 10^{-4}$ & 1.02 & $1 / F$ \\
$\mathbf{- 0 . 0 8}$ & -0.198 & $4.43 \times 10^{-4}$ & 1.04 & $1 / F$ \\
$\mathbf{- 0 . 0 6}$ & -0.178 & $9.66 \times 10^{-4}$ & 1.09 & $\sim 1 / F$ \\
$\mathbf{- 0 . 0 4}$ & -0.158 & $2.11 \times 10^{-3}$ & 1.21 & $1.2 / F$ \\
$\mathbf{0}$ & -0.118 & 0.01 & 2.02 & $2 / F$ \\
$\mathbf{0 . 1}$ & -0.018 & 0.49 & 98.52 & $98.5 / F$ \\
\hline
\end{tabular}

Table S1. For a quasi-reversible, one-step one electron reduction reaction, the dependence of a slope on $E-E^{0^{\prime}} . E^{0^{\prime}}=0.3 \mathrm{~V}, p=C_{O}{ }^{*} / C_{R}{ }^{*}=100, E_{e q}=0.418 \mathrm{~V}$. The shaded region shows a deviation of $(1+100 \mathrm{~b}) /(1-\mathrm{b})$ from unity. The potential range showing unity satisfies our K-L application. 


\begin{tabular}{ccccc}
\hline $\mathbf{E}-\mathbf{E}^{\mathbf{0}^{\prime}} / \mathbf{V}$ & $\mathbf{E}-\mathbf{E}_{\mathbf{e q}} / \mathbf{V}$ & $\mathbf{b}$ & $\mathbf{( b + 1 0 0 ) / ( \mathbf { b } - \mathbf { 1 } )}$ & slope \\
\hline $\mathbf{- 0 . 0 6}$ & 0.118 & 96.66 & 2.06 & $2 / F$ \\
$\mathbf{- 0 . 0 4}$ & 0.138 & 210.61 & 1.48 & $\sim 1.5 / F$ \\
$\mathbf{0}$ & 0.178 & $1.00 \times 10^{3}$ & 1.10 & $1.1 / F$ \\
$\mathbf{0 . 0 4}$ & 0.218 & $4.75 \times 10^{3}$ & 1.02 & $\sim 1 / F$ \\
$\mathbf{0 . 0 6}$ & 0.238 & $1.03 \times 10^{4}$ & 1.01 & $\sim 1 / F$ \\
$\mathbf{0 . 1}$ & 0.278 & $4.91 \times 10^{4}$ & 1.00 & $1 / F$ \\
$\mathbf{0 . 2}$ & 0.378 & $2.41 \times 10^{6}$ & 1.00 & $1 / F$ \\
$\mathbf{0 . 3}$ & 0.478 & $1.19 \times 10^{8}$ & 1.00 & $1 / F$ \\
$\mathbf{0 . 4}$ & 0.578 & $5.82 \times 10^{9}$ & 1.00 & $1 / F$ \\
\hline
\end{tabular}

Table S2. For a quasi-reversible, one-step one electron oxidation reaction, the dependence of a slope on $E-E^{0^{\prime}} . E^{0^{\prime}}=0.3 \mathrm{~V}, p=C_{R}{ }^{*} / C_{O}{ }^{*}=100, E_{e q}=0.122 \mathrm{~V}$. The shaded region shows a deviation of $(b+100) /(b-1)$ from unity. The potential range showing unity satisfies our K-L application.

In Figure S3, we schematically depicted an applicable potential range for the present K-L analysis in theoretical voltammograms for quasi reversible ET reactions with different $k^{0}$ and radius of UMEs. The grey shaded region represents an adaptable potential range. Figure S3(a) and S3(b) are for quasi reversible one electron reduction reaction with $k^{0}=4 \times 10^{-4} \mathrm{~cm} / \mathrm{s}$ and 0.4 $\mathrm{cm} / \mathrm{s}$ respectively. Figure S3(c) and S3(d) are for quasi reversible one electron oxidation reaction with $k^{0}=4 \times 10^{-4} \mathrm{~cm} / \mathrm{s}$ and $0.4 \mathrm{~cm} / \mathrm{s}$ respectively. Notably, the available range of potential becomes narrower as $k^{0}$ becomes faster. 
(a)
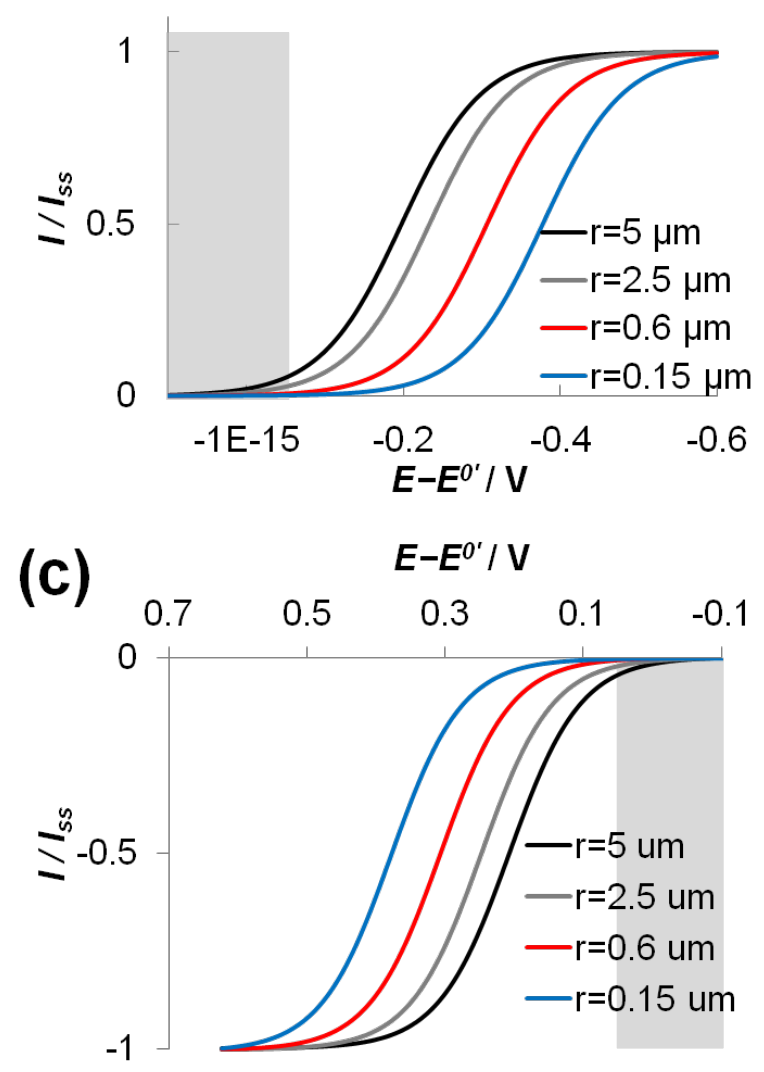

(b)

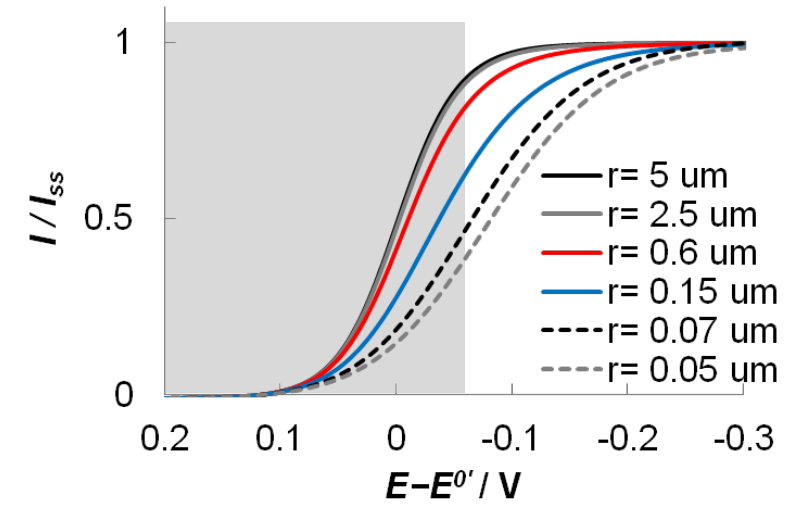

(d)

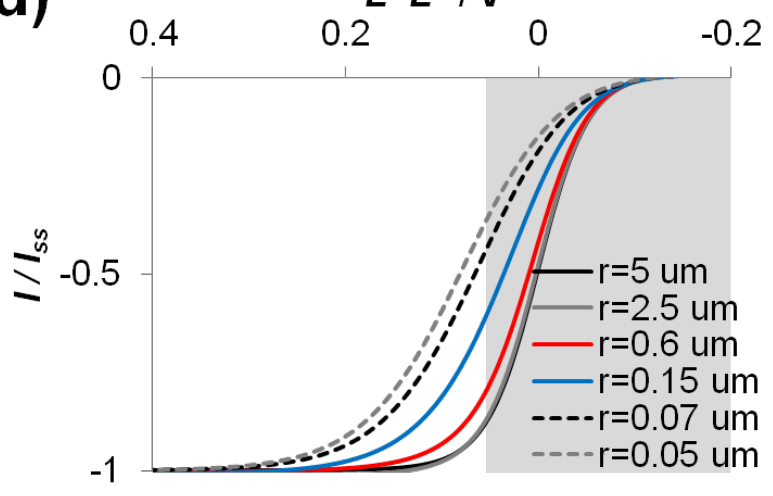

Figure S3. Schematic view of an applicable potential range for K-L analysis depicted in voltammograms of quasi reversible ET reactions. The grey shaded region represents an unadaptable potential range. (a) and (b) voltammograms of quasi reversible one electron reduction reaction with $k^{0}=4 \times 10^{-4} \mathrm{~cm} / \mathrm{s}$ and $0.4 \mathrm{~cm} / \mathrm{s}$ respectively with different radii of UMEs (from $5 \mu \mathrm{m}$ to 0.15 or $0.05 \mu \mathrm{m}$ ). (c) and (d) voltammograms of quasi reversible one electron oxidation reaction with $k^{0}=4 \times 10^{-4} \mathrm{~cm} / \mathrm{s}$ and $0.4 \mathrm{~cm} / \mathrm{s}$ respectively with different radii of UMEs (from $5 \mu \mathrm{m}$ to 0.15 or $0.05 \mu \mathrm{m}$ ).

\section{6) Finite Element Simulations}

Cyclic voltammograms were simulated by solving the corresponding 2D diffusion problems using the finite element method with COMSOL Multiphysics version 4.2a (COMSOL, Inc., Burlington, MA). In this work, the inlaid electrode was considered for the sake of simplicity. The model is given in Figure S4. All dimensions are in micrometer scale. Also, the parameters used for this simulation are given in Table S3. 


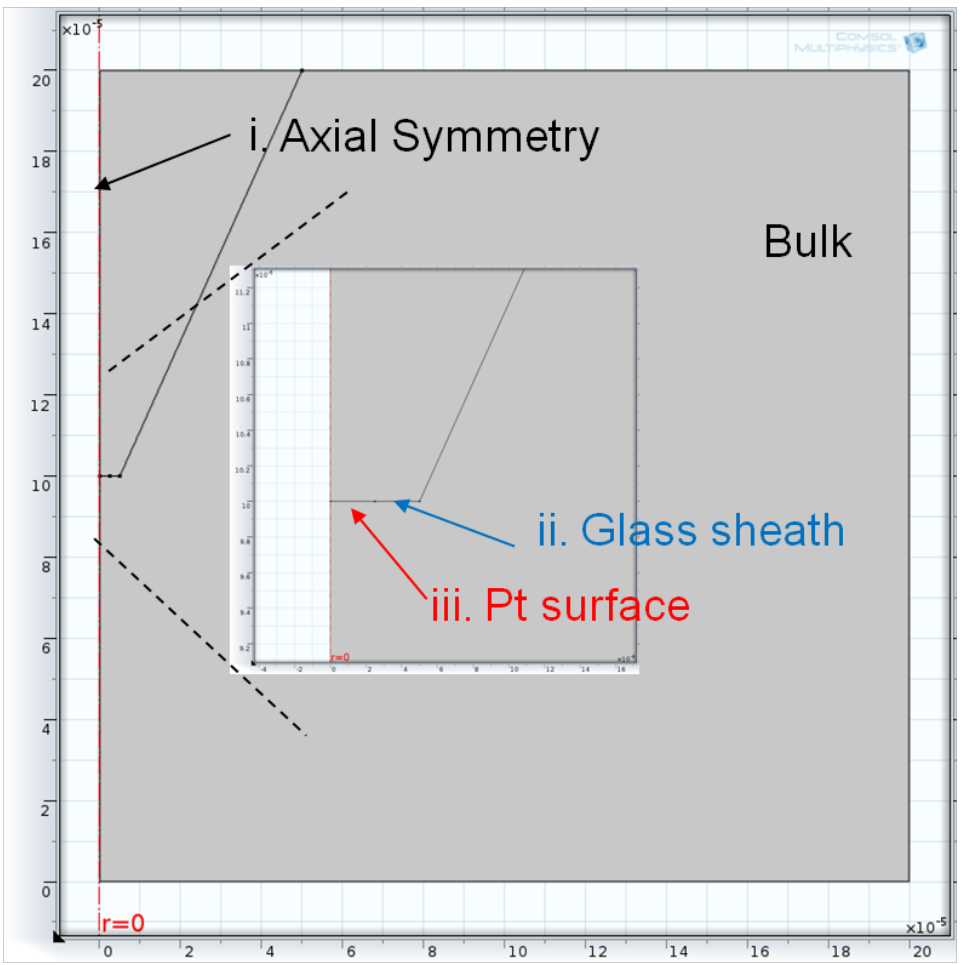

Figure S4. Geometry of 2D axial symmetric model used in simulations. Pt electrode disk was embedded in a glass sheath. All dimensions were in micrometer scale.

\begin{tabular}{|c|c|}
\hline parameters & values \\
\hline Diffusion coefficient, $D\left(\mathrm{~cm}^{2} / \mathrm{s}\right)$ & $7.4 \mathrm{E}-6$ \\
\hline Initial concentration of $\mathrm{O}, C_{O, \text { ini }}(\mathrm{mol} / \mathrm{L})$ & $1 \mathrm{E}-3$ \\
\hline Initial concentration of $\mathrm{R}, C_{R \text {, ini }}(\mathrm{mol} / \mathrm{L})$ & $1 \mathrm{E}-5$ \\
\hline Faraday constant, $F(\mathrm{C} / \mathrm{mol})$ & 96485 \\
\hline Number of transferred electrons, $n$ & 1 \\
\hline$n F / R T, f\left(\mathrm{~V}^{-1}\right)$ & 38.94 \\
\hline Standard reduction potential, $E^{0}(\mathrm{~V})$ & 0.3 \\
\hline Standard rate constant, $k^{0}(\mathrm{~cm} / \mathrm{s})$ & 0.4 \\
\hline Transfer coefficient, $\alpha$ & 0.5 \\
\hline Scan rate, $v(\mathrm{~V} / \mathrm{s})$ & 0.02 \\
\hline Initial potential, $E_{\text {ini }}(\mathrm{V})$ & 0.6 \\
\hline Final potential, $E_{\text {fin }}(\mathrm{V})$ & -0.3 \\
\hline
\end{tabular}

Table S3. Parameters used in the simulations 
S1) Hills, C.M..; Kim, J.; Bard. A.J., J. Am. Chem. Soc., 2015, 137, 11321-11326.

S2) Bard, A.J.; Faulkner, L.R., Electrochemical Methods, Wiley, New York, 1980 